\title{
Septic shock, acute renal and liver failure following unsafe abortion using bitter leaves and wandering jew in northern Uganda: a case series
}

\author{
Gasthony Alobo ${ }^{1}$, Cristina Reverzani ${ }^{2}$, Violah Nahurira ${ }^{2}$, and Laura Sarno ${ }^{3}$ \\ ${ }^{1}$ Lira University \\ ${ }^{2}$ St. Mary's Hospital Lacor \\ ${ }^{3}$ University of Naples Federico II
}

February 25, 2022

\begin{abstract}
This case series describes the unusual severe toxicity of two herbal medicines that are frequently used to induce abortion in Northern Uganda. It highlights possible associations of Commelina Africana (wandering jew) with uterine necrosis complicated by and sepsis, and of Vernonia amygdalina (bitter leaf) with acute liver and renal failure

Septic shock, acute renal and liver failure following unsafe abortion using bitter leaves and wandering jew in northern Uganda: a case series.

Gasthony Alobo ${ }^{1,3}$, Cristina Reverzani ${ }^{3}$, Violah Nahurira ${ }^{3}$, Laura Sarno ${ }^{2 *}$ Correspondence: gasthonya@gmail.com

${ }^{1}$ Department of Obstetrics and Gynaecology, Lira University, Lira, Uganda

${ }^{2}$ Department of Neurosciences, Reproductive Science and Dentistry, University of Naples Federico II, Via Pansini 5, 80131, Naples, Italy.

${ }^{3}$ Department of Obstetrics and Gynaecology, St. Mary's Hospital Lacor, P.O. Box 180 Gulu, Uganda.

Corresponding author: Dr. Alobo Gasthony, e-mail: gasthonya@gmail.com
\end{abstract}

\begin{abstract}
Background: Unsafe abortion is a major problem in Uganda, being one of the leading causes of maternal morbidity and mortality. An estimated 297,000 unsafe abortions are performed yearly, with an abortion rate of 54 per 1,000 women aged 15-49; nearly 85,000 women have been treated annually for complications of unsafe abortion, leading to about 1,200 deaths. Abortions are performed mostly under unsafe conditions, by people without medical training. In rural areas in northern Uganda, women often resort to traditional providers, who use local herbs as abortion remedies. Little is known about the biological properties of these herbs and their toxicity profile.

Case presentation : Here we present the case series of two women, of 31 and 24 years of age, who underwent unsafe abortion for unintended pregnancy by using herbal medicines, i.e. Commelina Africana (wandering jew) and Vernonia amygdalina (bitter leaf) respectively. While the first case resulted in uterine necrosis and pelvic peritonitis, which required multiple surgical interventions and the use of reserve antibiotics, the second case resulted in liver and renal failure that led to the death of the patient.
\end{abstract}


Conclusion: This case series describes the unusual severe toxicity of two herbal medicines that are frequently used to induce abortion in Northern Uganda. It highlights possible associations of Commelina Africana (wandering jew) with uterine necrosis complicated by pyometra and sepsis, and of Vernonia amygdalina(bitter leaf) with acute liver failure and renal failure.

Keywords: Unsafe abortion, herbal medicines, herbs, northern Uganda, case series

\section{Background}

Worldwide, the leading causes of maternal morbidity and mortality are direct obstetric complications and include hemorrhage, sepsis, hypertensive disorders, and abortion; the latter is responsible for $7.9 \%$ of maternal deaths globally and 9.6\% in Sub-Saharan Africa (SSA) [1]. The World Health Organization defines unsafe abortion as a procedure for terminating a pregnancy performed by people lacking the necessary skills or in an environment, not in conformity with minimal medical standards, or both [2].

In Uganda, although the official data of the Ministry of Health indicate that abortion accounts for $5 \%$ of maternal deaths [3], the mortality due to abortion complications could be higher due to restrictive laws. It is estimated that 297,000 unsafe abortions are performed yearly, with an abortion rate of 54 per 1,000 women aged 15-49. Nearly 85,000 women have been treated annually for complications of unsafe that include incomplete abortion, sepsis, and uterine perforations [4] making it the main gynecologic cause of admission, leading to about 1,200 deaths annually [5].

The abortion rate is even higher in northern Uganda (70 per 1,000 women aged 15-49 years) [4], a predominantly rural region that is still recovering from a 20-year conflict that left many people displaced from their homes and economically disadvantaged. One of the factors contributing to the high number of abortion complications is the unmet need for contraception, which is still very high (28\%). Contraceptive use in Uganda remains very low; only $17 \%$ of all women of reproductive age, and $18 \%$ of married women, utilize modern contraception [6]. This has resulted in a high total fertility rate (TFR) of 5.4 [7]. It is estimated that almost half of the pregnancies in Uganda are unintended, with $15 \%$ of the total pregnancies ending in unsafe abortion and constituting nearly one-third of the maternal deaths among the country's young people [8]. The situation is even more dramatic among rural, poor, and less educated women, such as in the northern region.

Legal provisions for abortion in Uganda are very restrictive. Art. 22(2) of the Constitution of Uganda (1995) quotes: "No person has the right to terminate the life of an unborn child except as may be authorized by law ". The Penal Code Act (Cap. 120), as amended through the Penal Code Amendment Act (No. 8 of 2007), Section 205, allows only abortion if "carried out in good faith to preserve the mother's life" . Therefore, safe 'legal' abortion is not available and accessible, except for rare cases where it is the only way to save a woman's life, and many healthcare providers prefer not to offer safe abortion to women who need it [9]. It is known that highly restrictive laws do not reduce abortion rates, but rather increase the likelihood of unsafe abortion [10]. Consequently, in a country like Uganda where abortion is highly stigmatized [11, 12], many women seek illegal abortion with unsafe traditional methods, offered by non-medical providers under unsafe conditions. While in urban areas the use of prostaglandin analogs is the most common method of unsafe abortion, in rural areas women resort to the intra-vaginal use of sharp objects (safety pins, nails, knives or sticks), to the ingestion of caustic substances (detergents or bleach) and more often to the use of local herbs, such as Commelina Africana and Vernonia amygdalina, which are readily available in the countryside [13].

In this small case series, we described two cases of unsafe abortions induced with very common herbs in northern Uganda, which ended up with severe complications and, in one case, led to the death of the woman.

\section{Case presentation:}

\section{Case 1}

N.A., a 31-year old female, para 2, abortus 1, at 5 weeks of amenorrhea and HIV negative, was admitted to our Gynecology ward via the Emergency Department. The patient presented with a 2-week history of lower 
abdominal pain, 3 days of vomiting, and 2 days of loose stools. She did not report any bleeding or discharge per vagina; the micturition habit was normal. She reported not being pregnant in recent months and that the symptoms started spontaneously, without any identifiable cause, and worsened progressively.

At admission, the patient was conscious, with Glasgow Coma Scale (GCS) of 15/15, but pale and in obvious discomfort; her blood pressure $(\mathrm{BP})$ was $96 / 56 \mathrm{mmHg}$, pulse rate (PR) was 112 beats per minute and temperature was $38.7^{\circ} \mathrm{C}$. Abdominal examination revealed severe distension, generalized tenderness, and guarding; bowel sounds were present. The vaginal examination demonstrated no active bleeding but moderate foul-smelling discharge; the cervical os was closed. At the bimanual examination, the uterus was bulky, with severe cervical motion tenderness. The physical examination of the other systems was unremarkable.

Her blood investigations showed hemoglobin $(\mathrm{Hb})$ of $6.5 \mathrm{gm} / \mathrm{dl}$, white cell count of $32.3 \times 10^{9} / 1$, platelets of $105 \times 10^{9} / 1$. The urine human chorionic gonadotropin (hCG) quick test was positive. A pelvic ultrasound (US) scan showed an empty and bulky uterus, normal-looking ovaries, and the presence of an echogenic pelvic mass; a diagnosis of a pelvic abscess was made and an exploratory laparotomy planned, after resuscitation of the patient. During the exploratory laparotomy, the uterus was severely necrotic and there was pus collection of 2 litres in the paracolic gutters and the sub-diaphragmatic recess.

During the postoperative period, antibiotic therapy with intravenous first line broad-spectrum antibiotics was performed, but the patient remained febrile and started discharging pus from the laparotomy wound on the $5^{\text {th }}$ postoperative day. A wound swab culture was performed, revealing a multi-resistant Escherichia coli, sensitive to Gentamicin and Chloramphenicol. Therapy with a combination of these antibiotics did not show any improvement in the following days and, on the $15^{\text {th }}$ postoperative day, the incision site started gaping, with copious pus discharge.

A further pelvic ultrasound scan showed an echo-complex fluid collection in the pouch of Douglas, with a volume of around $265 \mathrm{ml}$; the findings were in line with a pelvic abscess. A repeat laparotomy confirmed the diagnostic hypothesis and an extensive pelvic lavage was done. The patient was treated postoperatively with a reserve antibiotic (intravenous Meropenem) and after 10 days was discharged home in good general conditions, on oral antibiotics. At discharge from the hospital, the patient reported having induced the abortion with a local herb, known as "wandering jew", three weeks before admission, by inserting some pieces of the stem of the plant in the vagina; a few days after the expulsion of the fetus, she started developing lower abdominal pain and abdominal distension, associated with per vagina foul-smelling discharge.

\section{Case 2}

A.E., a 24-year-old female, gravida 4, para 3, at unknown weeks of amenorrhea, was admitted to our Gynecology ward with a history of fever and chills, general body weakness, tea-colored urine and cramping lower abdominal pain, radiating to the waist, that started spontaneously and increased progressively in intensity. There was no history of vomiting or nausea, vaginal bleeding, or discharge; the patient reported normal micturition and bowel habits. The history of complaints of other systems was unremarkable.

The patient, who was married with three living children, did not attend antenatal care for the current pregnancy; obstetric and gynecological histories were unremarkable. There was no history of chronic illnesses, while the HIV status was unknown. The patient denied the use of herbal medicine or any other medication in this pregnancy.

On physical examination, she was sick looking, with moderate pallor, severe jaundice with dry mucous membranes, dry skin, and no edema. Her BP was $93 / 61 \mathrm{mmHg}$, with PR of 94 beats per minute and a temperature of $36.8^{\circ} \mathrm{C}$.

Abdominal examination revealed a normal fullness, with a moderate tenderness in the hypogastric region; no organomegaly or masses were palpable. On per vagina exam, vulva and vagina were normal, the cervical os was closed and there was no active bleeding or discharge. The physical examination of the other systems was unremarkable. 
Her investigations showed a positive urine hCG quick test, while the blood smear did not evidence any malaria parasites. The complete blood count showed a white cell count of $28.1 \times 10^{9} / \mathrm{L}$, with granulocytes of $20.5 \times 10^{9} / \mathrm{L}, \mathrm{Hb}$ of $11.7 \mathrm{~g} / \mathrm{dl}$, and red blood cells of $3.66 \times 10^{12} / \mathrm{L}$, platelets of $270 \times 10^{9} / \mathrm{L}$ and negative hepatitis B surface antigen test. Liver function: Alanine transferase of 1,796U/L (0-40), Aspartate transferase of 1,628U/L (0-37), Alkaline phosphatase of $159 \mathrm{U} / \mathrm{L}$ (98-279) and albumin of 2.5g/dl (3.8-5.1). Renal function test: urea of $82 \mathrm{mg} / \mathrm{dl}(10-55)$, creatinine of $7.1 \mathrm{mg} / \mathrm{dl}(0-1.3)$ and urea/creatinine ratio of 11.54 . Serum electrolytes: sodium of $128.2 \mathrm{mmol} / \mathrm{L}$ (134-146), potassium of $4.6 \mathrm{mmol} / \mathrm{L}$ (3.5-5.5) and chloride of $105.9 \mathrm{mmol} / \mathrm{L}(98-108)$.

A pelvic ultrasound scan showed a gravid uterus with an intrauterine fetus in cephalic presentation, but no cardiac activity was present; the amniotic fluid was reduced and the internal cervical os was open. Moreover, the left maternal kidney was mildly enlarged and echogenic, with normal margins. The findings of the ultrasound scan were in line with a missed abortion at 14 weeks of gestation and maternal left nephropathy. Our impression was a para 3, abortus 1 , with missed abortion at 14 weeks of gestation, acute hepatitis, and nephropathy. The patient was stabilized and managed conservatively, without relevant improvement.

On the $2^{\text {nd }}$ day of hospital stay, there was a new complaint of bleeding per vagina; the patient was still sick looking, afebrile, with moderate pallor and deep jaundice. Her BP was 110/65 mmHg, with PR of 69 beats per minute and random blood sugar (RBS) of $3.4 \mathrm{mmol} / \mathrm{L}$. On per vagina examination, the cervical os was still closed but fresh blood was found on the examining finger. The patient was treated with dextrose, lactulose, and broad-spectrum antibiotics; after the administration of one dose of Misoprostol, the fetus and the placenta were expelled. Thereafter, the patient was restless, although the vital parameters were in the normal range.

On the $3^{\text {rd }}$ day of hospital stay, the patient became lethargic, with slurred speech and restless, moderately pale, and increasingly jaundiced. Her BP was $119 / 73 \mathrm{mmHg}$, with PR of 69 beats per minute, temperature of $36.7^{\circ} \mathrm{C}$ and RBS of $4.5 \mathrm{mmol} / \mathrm{l}$. GCS was $12 / 15(\mathrm{E}=4, \mathrm{M}=5, \mathrm{~V}=3)$, with pupils equal and reactive to light, normal tone, and reflexes. The patient was in mild respiratory distress, with a respiratory rate of 28 breaths/minute and oxygen saturation of $95 \%$ on room air. The urine was greenish, with a urine output of $300 \mathrm{ml} / 24 \mathrm{hrs}$. A nasogastric tube was inserted and drained $200 \mathrm{ml}$ of dark brown colored fluids. The patient was lethargic and disoriented to time; a diagnosis of hepatic encephalopathy grade 2, based on West Haven criteria, was made and the plan was to continue the conservative therapy. On the early morning of the $4^{\text {th }}$ day of hospital stay, the patient passed away.

Only after her death, a collateral history could be obtained. The patient's husband, who wanted more children, wasn't aware that his wife was pregnant. The couple had previously argued on this issue since the patient was not willing to face a new pregnancy, but the husband prohibited her from using any modern contraceptive methods. As reported by the mother in law, when the patient realized that she was pregnant, she consulted a traditional healer, who provided her a local herb known as "bitter leaf".

\section{Discussion and conclusion:}

In Uganda, medicinal plants are widely used by indigenous ethnic groups, both as food vegetables and culinary herbs and in traditional medicine. In a study conducted in western Uganda, 75 plants commonly used to induce labor have been identified; most of them possess uterotonic properties and, at unspecified high doses, their toxicity may be life-threatening for both the mother and the fetus [14].

In northern Uganda, two plants are commonly used to induce labor, i.e. the Commelina Africana, also known as "wandering jew" (Figure 1), and the Vernonia amygdalina, also known as "bitter leaf" (Figure 2). The uterotonic properties of these herbs have been already tested and confirmed [15]. Commelina Africana (" Wandering jew"; "Lototo" in Acholi language and "Otoro" in Luo language), is known to have hypoglycemic effects [10], as well as uterotonic activity; in fact, it can increase both the force of contractions and their frequency, with a dose-response curve [15]. To induce abortion, the stem of the plant is normally broken in small pieces and inserted in the vagina, adjacent to the cervix, to induce it to dilate [14]. The severe complications due to the use of this herb, as reported in Case 1, seem to be due to its insertion in the vagina 
under unsterile conditions, which allow the ascension of pathogens to the pelvic cavity passing through the cervix, the uterus and the fallopian tubes, causing massive pelvic abscesses, severe peritonitis, and septic shock. In our case, this condition required multiple surgical interventions with extensive abdominal lavage, as well as the use of reserve antibiotics.

Vernonia amygdalina ("Bitter leaf"; "Labwori" in the Acholi language and "Olusia" in Luo language) is an indigenous shrub that grows in most parts of sub-Saharan Africa and may be found growing wild along the edges of agricultural fields. It grows to a height of 1-2 m, with elliptical leaves up to $20 \mathrm{~cm}$ long. It is commonly called "bitter leaf" in English because of its bitter taste. As reported by Echemet al.[16], Vernonia amygdalina plays a big role in the diet of indigenous people throughout equatorial Africa because of the presence of vitamins and mineral salts [15]. For numerous African ethnic groups, it is also considered a very strong medicine in the treatment of malarial fever, schistosomiasis, amoebic dysentery, and several other intestinal parasites and stomach aches; extracts thereof have been also used traditionally in the treatment of sexually transmitted diseases, cough, constipation, and hypertension. Its ability to contract the uterus, thereby inducing abortion, has been validated [15]. Vernonia species contain phytochemical principles, which include bitter sesquiterpene lactones, vernolepin, vernodalin, vernomygdin, and steroid glucosides, from which its biological properties were derived [17]. Vernonia amygdalina,especially those grown along heavy traffic routes, can concentrate high levels of heavy metals such as Iron ( $\mathrm{Fe})$, Manganese ( $\mathrm{Mn}$ ), Lead (Pb), chromium $(C r)$, Zinc $(Z n)$ and Cadmium $(C d)$; which have the potential to act as biological poisons even at parts per billion levels, [16]. The concentration of these metals is highest in the leaves, which are commonly used for decoctions.

In case 2, the use of Vernonia amygdalina led to complications requiring admission to the hospital; the patient presented with reduced GCS due to hepatic encephalopathy, associated with other signs of acute renal and liver failure. Typical laboratory findings were hypoglycemia, leucocytosis, and elevated liver enzymes, associated with elevated creatinine and urea values. The course of the disease was deadly.

In conclusion, we reported two cases describing the unusual severe toxicity of two herbal medicines that are frequently used to induce abortion in northern Uganda. In particular, the use of Commelina Africana may be associated with pyometra and/or pyoperitoneum and sepsis, while Vernonia amygdalina may lead to acute liver and renal failure, which can be fatal.

The ingestion of medicinal plants to induce abortion involves the risk of severe maternal morbidity and mortality; patients should be informed that the use of these medicinal plants as abortifacients can be lifetreating. Active policy to improve knowledge of contraception and family planning is required.

Moreover, further field and laboratory research is needed to identify the active principles contained in such herbal medicines and their biological properties, to achieve a better understanding of their pathophysiology, dosage levels, and toxicity. This would help in the treatment of the associated complications and the development of possible antidotes.

\section{Declarations:}

\section{List of abbreviations:}

BP: blood pressure; GCS: Glassgow coma scale; Hb: hemoglobin; hCG: human chorionic gonadotropin; HIV: human immunodeficiency virus; PR: pulse rate; SSA: sub-Saharan Africa; TFR: total fertility rate.

\section{Ethical approval and consent to participate:}

Ethical approval was provided by the Lacor Hospital Institutional Research Ethics Committee. Before making this case series, written informed consent was obtained from the patient (case 1) and the next of kin of the deceased patient (Case 2). The patients' identities were duly concealed.

\section{Consent for publication:}


Written informed consent for publication of this case series and the accompanying images was obtained from the patient (Case 1) and the next of kin of the deceased patient (Case 2).

\section{Availability of data and materials:}

All data generated or analyzed during this study are included in this manuscript.

\section{Competing interests:}

The authors declare no conflicts of interest.

\section{Funding:}

There was no external funding to support this case report.

\section{Authors' contribution:}

CR and GA wrote the case report Background and Discussion; VN collected the information on the patients and compiled the Case Presentation; LS made the final review and editing of the manuscript before submission. All authors have read and approved the manuscript.

\section{Acknowledgments:}

We would like to acknowledge the entire staff of the gynecology ward at Lacor Hospital, especially the nurses who attended the patients during their hospital stay. We would like to thank the patients' next of kin, who consented to the preparation and publication of this case series. We finally acknowledge the Mother-Child Health Lacor and South Sudan (MoCHeLaSS) project, which facilitated the training of some team members in research methods.

\section{References:}

1. Say, L., et al., Global causes of maternal death: a WHO systematic analysis. The Lancet Global Health, 2014. 2 (6): p. e323-e333.

2. Ganatra, B., et al., From concept to measurement: operationalizing WHO's definition of unsafe abortion . 2014, SciELO Public Health.

3. Ministry of Health, The Annual Health Sector Performance Report 2018/2019. Kampala: Republic of Uganda,2019.

4. Singh, S., et al., The incidence of induced abortion in Uganda. International family planning perspectives, 2005: p. 183-191.

5. Mulumba, M., et al., Access to safe abortion in Uganda: leveraging opportunities through the harm reduction model.International Journal of Gynecology \& Obstetrics, 2017.138 (2): p. 231-236.

6. Musoke, J.T. and B. Tolboom, Uganda Country Report - Country Needs Assessments on Safe Abortion Advocacy. FIGO and Uganda Obstetrical ans Gynaecological Society AOGU, 2018.

7. Uganda Bureau of Statistcs (UBOS) and ICF, Uganda Demographic and Health Survey 2016: Key Indicators Report . 2017, UBOS and Rockville: Kampala, Uganda and Maryland, USA.

8. Hussain, R., Unintended pregnancy and abortion in Uganda.Issues in Brief (Alan Guttmacher Institute), 2013(2): p. 1-8.

9. Paul, M., et al., Barriers and facilitators in the provision of post-abortion care at district level in central Uganda-a qualitative study focusing on task sharing between physicians and midwives. BMC health services research, 2014. 14 (1): p. 28.

10. Singh, S., et al., Abortion worldwide 2017: uneven Progress and unequal Access Abortion worldwide 2017: uneven Progress and unequal Access. 2018. 
11. Larsson, S., et al., The discourses on induced abortion in Ugandan daily newspapers: a discourse analysis. Reproductive health, 2015. 12 (1): p. 58.

12. Cleeve, A., et al., Abortion as agentive action: reproductive agency among young women seeking postabortion care in Uganda. Culture, health \& sexuality, 2017. 19 (11): p. 1286-1300.

13. Kiguba, R., et al., Herbal medicine use and linked suspected adverse drug reactions in a prospective cohort of Ugandan inpatients.BMC complementary and alternative medicine, 2016. 16 (1): p. 145.

14. Kamatenesi-Mugisha, M. and H. Oryem-Origa, Medicinal plants used to induce labour during childbirth in western Uganda. Journal of ethnopharmacology, 2007. 109 (1): p. 1-9.

15. Rasch, V., et al., Unsafe abortion in rural Tanzania-the use of traditional medicine from a patient and a provider perspective. BMC pregnancy and childbirth, 2014. 14 (1): p. 419.

16. Echem, O.G. and L. Kabari, Heavy metal content in Bitter Leaf (Vernonia amygdalina) grown along heavy traffic routes in Port Harcourt. Agricultural Chemistry, 2013: p. 201-210.

17. Clement, E. et al., Significance of Bitter Leaf (Vernonia Amagdalina) In Tropical Diseases and Beyond: A Review . Malaria Chemotherapy Control and Elimination, 2014. 3 (1): p. 1-10.

Figure Legends: 


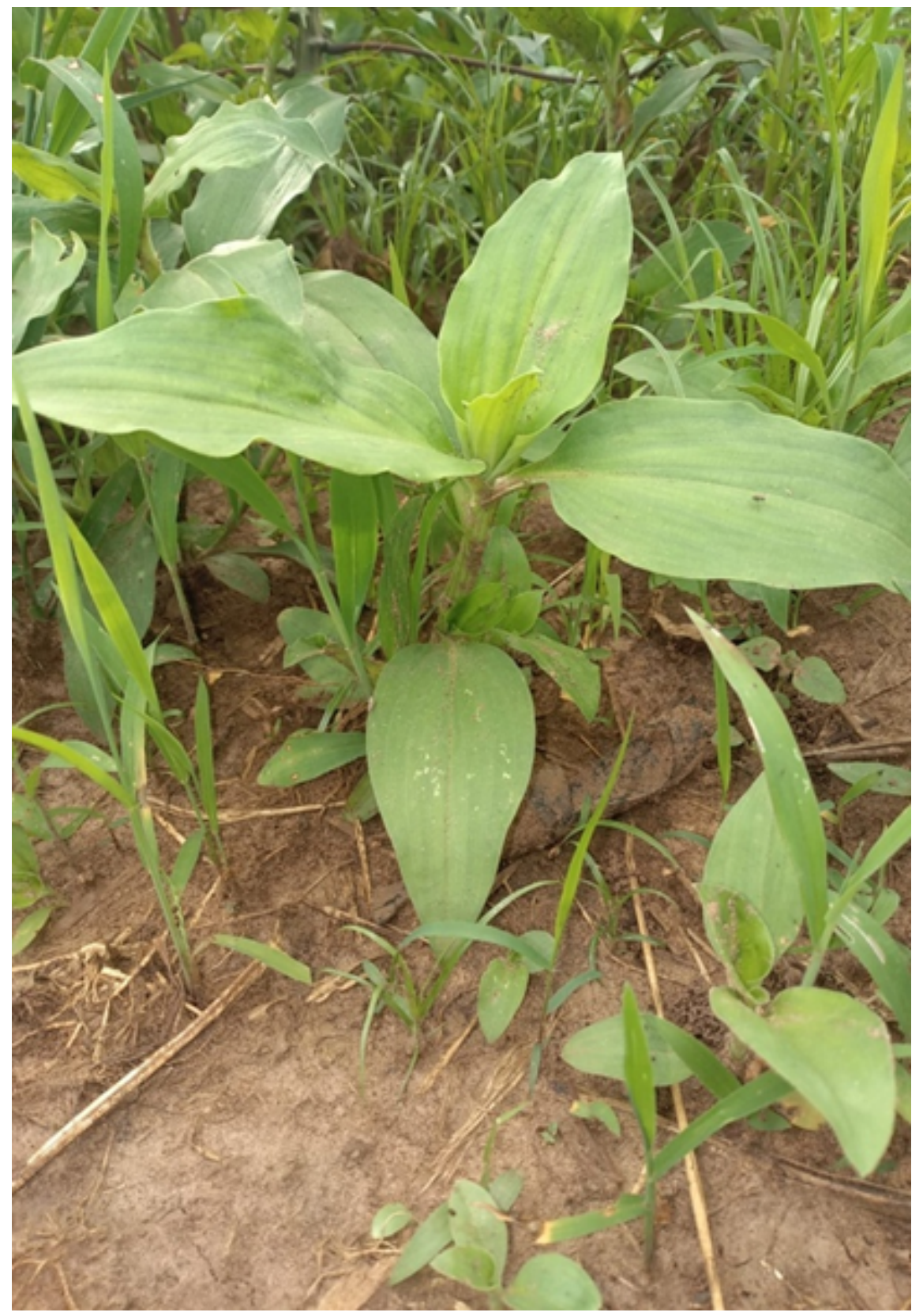

Figure 1 - Commelina Africana or "wandering jew"

Figures 1 and 2 are photographs of the herbs that were reportedly used by the two cases following lead by the traditional herbalists who administered them. The photographs were taken by the authors, CR and GA. 


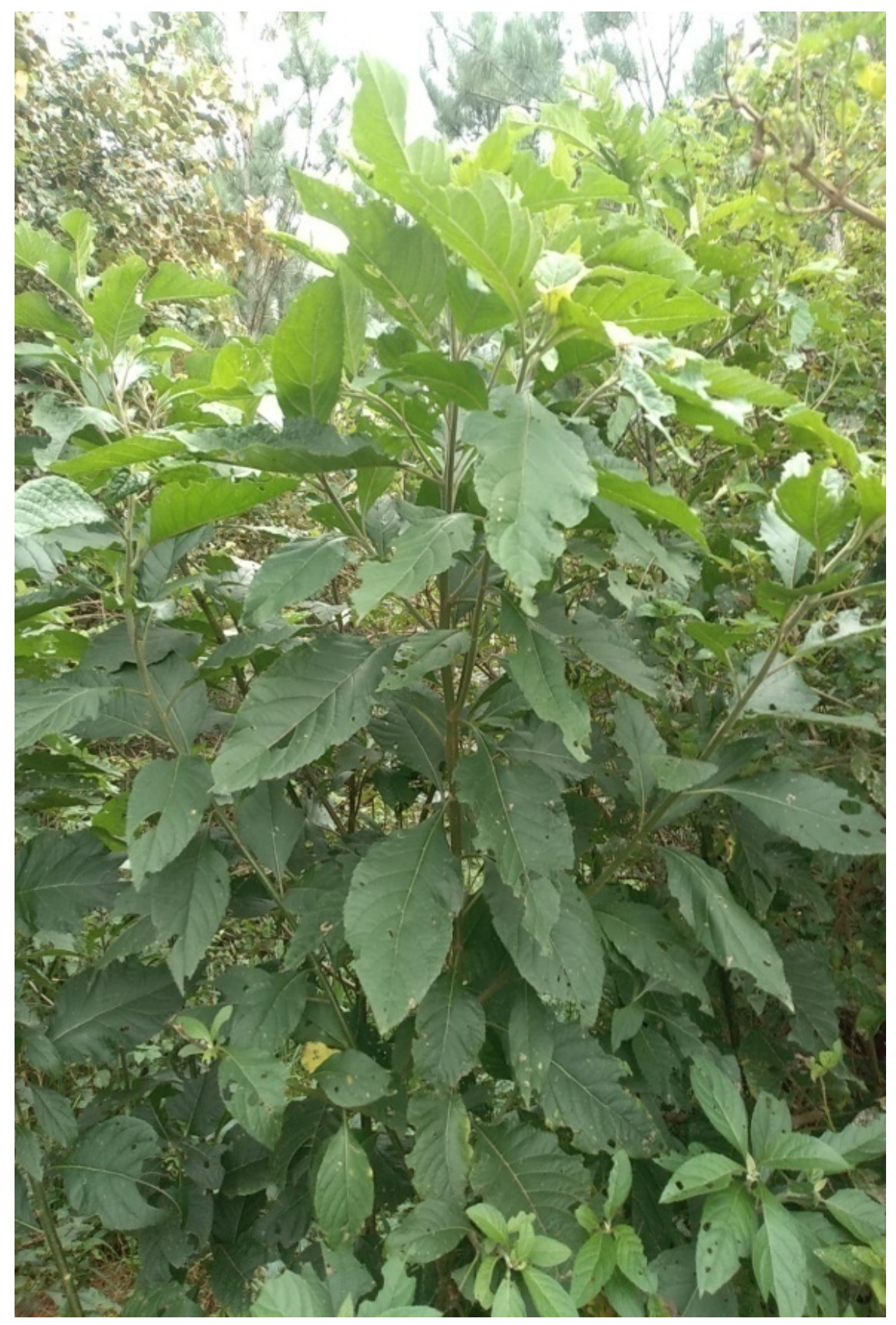

Figure 2 - Vernoniaamygdalina or "bitter leaf" 